\title{
Baseline in vivo, ex vivo and molecular responses of Plasmodium falciparum to artemether and lumefantrine in three endemic zones for malaria in Colombia
}

\section{Samanda Aponte ${ }^{a, 1}$, Ángela Patricia Guerra ${ }^{a, b, *}$, Catalina Álvarez-Larrotta $^{a}$, Sindy Durley Bernal ${ }^{a}$, César Restrepo ${ }^{a}$, Camila González ${ }^{b}$, María Fernanda Yasnot ${ }^{c}$ and Angélica Knudson-Ospina ${ }^{d}$}

\begin{abstract}
${ }^{a}$ Grupo de Bioquímica y Biología Celular, Instituto Nacional de Salud, Bogotá, D.C., Colombia; ${ }^{b}$ Grupo de Parasitología, Dirección de Redes en Salud Pública, Instituto Nacional de Salud, Bogotá, D.C., Colombia; ' Grupo de Investigaciones Microbiológicas y Biomédicas de Córdoba-GIMBIC, Universidad de Córdoba, Montería, Colombia; ${ }^{d}$ Departamento de Microbiología, Facultad de Medicina, Universidad Nacional de Colombia, Bogotá, D.C., Colombia

\footnotetext{
${ }^{1}$ Present address: Unidad de Parasitología, Departamento de Salud Pública, Facultad de Medicina, Universidad Nacional de Colombia, Bogotá, D.C., Colombia

*Corresponding author: Tel: +57(1) 2207700 ext. 1337; E-mail: aguerra@ins.gov.co
}

Received 29 November 2016; revised 23 March 2017; editorial decision 24 March 2017; accepted 13 April 2017

\begin{abstract}
Background: Colombia began using artemisinin-based combination therapies for the treatment of uncomplicated Plasmodium falciparum malaria in 2006. It is necessary to implement resistance surveillance to antimalarial drugs in order to promptly detect changes in parasite susceptibility. The aim of this study was to establish a susceptibility baseline of $P$. falciparum to artemether-lumefantrine using three monitoring tools.
\end{abstract}

\begin{abstract}
Methods: Patients with uncomplicated malaria treated with artemether-lumefantrine underwent clinical and parasitological follow-up over 28 days. Ex vivo test was performed using the microtest technique for chloroquine, arthemeter, dihydroartemisinin and lumefantrine. Pfmdr1 copy number and polymorphisms in Pfk13, Pfatp6, Pfcrt and Pfmdr1 genes were analyzed.

Results: From a total of 150 screened patients, 49 completed follow-up for 28 days. All treated patients had adequate clinical and parasitological responses. Parasitic clearance showed a drastic reduction of parasite biomass at 24 hours and complete elimination at 48 hours. One hundred eleven isolates were processed, all exhibited high susceptibility to artemisinins and a slight decrease in susceptibility to lumefantrine. No genetic polymorphisms associated with resistance to artemisinin were found.

Conclusion: This study generated a susceptibility baseline in response to therapy with Coartem (artemetherlumefantrine) with numerical reference values, which will allow data comparison with future studies to systematically monitor changes in the parasite and to provide an early alert to the health authorities.
\end{abstract}

Keywords: Artemisinin-based combination therapies (ACT), Colombia, Plasmodium falciparum, Polymorphisms, Resistance surveillance, Therapeutic efficacy studies (TES)

\section{Introduction}

The current goal of the WHO Global Technical Strategy for Malaria, 2016-2030 is to promote elimination of the disease, especially in low transmission areas where resistance to antimalarial drugs has commonly originated. ${ }^{1}$ Despite the worldwide reduction of malaria incidence and the number of fatal cases registered in recent years, in the Greater Mekong subregion where multidrug resistance has historically emerged, therapeutic efficacy failures with artemisinin-based combination therapies (ACT), such as artesunate-mefloquine, have increased. ${ }^{2}$ This situation, so far unique to this area, has generated an epidemiological alert on the global commitment to preserve the effectiveness of artemisinin as a treatment for Plasmodium falciparum malaria.

In order to tackle the growing drug resistance, WHO has recommended the implementation of regular surveillance programmes employing tools such as therapeutic efficacy studies (TES), ${ }^{3}$ ex vivo assays and molecular markers, to facilitate monitoring and containment. ${ }^{4}$ Additionally, it has been shown that measurement of the parasitemia at 72 hours post-treatment with ACTs provides a 


\section{S. Aponte et al.}

simple and helpful screening method to detect changes in the pattern of parasite susceptibility to artemisinins, ${ }^{5}$ becoming currently one of the most widely used tests.

In Colombia, the Ministerio de Protección Social implemented the use of ACTs in 2006. However, in 2007 antimalarial therapies were unified with artemether-lumefantrine (ART-LUM) as the adopted combination, and it still remains so today. ${ }^{6}$ Since the introduction of ACTs in Colombia, studies have shown high efficacy with artesunate-mefloquine ${ }^{7}$ and artesunate-amodiaquine, ${ }^{8}$ and low inhibitory concentrations 50 ( $\mathrm{IC}_{50}$ ) for artemisinin derivatives, such as artesunate ${ }^{9}$ and dihydroartemisinin ${ }^{10}$ (DHA) and partner drugs as lumefantrine (LUM), ${ }^{10}$ suggesting appropriate responses to ACTs. However, little information is available on the therapeutic efficacy of ART-LUM combination or changes in the parasites' susceptibility or emergence of polymorphism associated with artemisinins and lumefantrine resistance.

The aim of this study was to establish the behavior baseline of $P$. falciparum populations circulating in Colombia and patients' response to treatment, using the tools available for monitoring the ART-LUM scheme in three sentinel sites. This response baseline to Coartem (artemether-lumefantrine) in Colombia will be the point of reference for subsequent measurements, allowing data comparison and giving information about necessary future changes.

\section{Materials and methods}

\section{Geographic area and study population}

Three sentinel sites were selected, taking into account the incidence of P. falciparum malaria in Colombia from 2009 to 2012. The sites were radically different in terms of the epidemiology and transmission of $P$. falciparum; one site was set up in the southern part of the Pacific region (Nariño), another was established in the Atlantic region (Córdoba), and the third one in southeastern Colombia (Guaviare) (Figure 1).

\section{Therapeutic efficacy study}

Sample size was determined according to an expected proportion of $5 \%$ treatment failures in a population of infinite size, with a $95 \%$ confidence interval and $+4 \%$ accuracy. A total of 150 patients were included given the likelihood of a $20 \%$ patient loss in a 28-day study. Since the epidemiological behavior of malaria is different in the three chosen departments, the sample size was proportional to the number of expected $P$. falciparum cases by locality; the estimated number of patients to be included was therefore 38, 102 and 10 for Córdoba, Nariño and Guaviare, respectively.

The selection of patients was conducted in five medical centers or diagnostic facilities belonging to the public hospital network. The selected patients met the following inclusion criteria: $1 . \geq 2$ years of age; 2. P. falciparum mono-infection and uncomplicated malaria; 3.

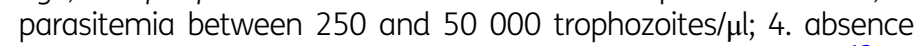
of chloroquine in the urine (negative Saker-Solomons test): ${ }^{13} 5$. non-pregnant women with a negative pregnancy test (Xerion-Le Belle, IMEX group, Bogotá, Colombia), 6. patients without any concomitant chronic illness. Patients with clinical evidence of severe malaria were excluded. After the diagnosis by thick blood smears

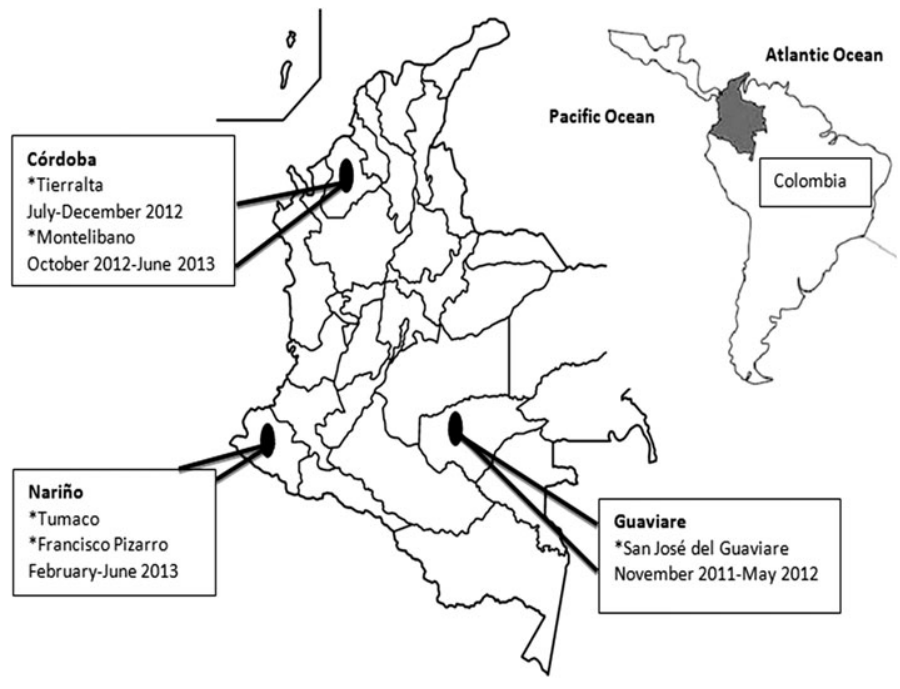

Figure 1. Sentinel sites selected to carry out the study. Three sentinel sites were chosen: one in Nariño department, which presents an annual parasitaemia index (API=10.07) and an annual P. falciparum index (AFI=9.58). ${ }^{11}$ In this department the samples were collected in two municipalities: Tumaco (lat $1^{\circ} 49^{\prime} \mathrm{N}$, long $78^{\circ} 52^{\prime} \mathrm{W}$ ) and Francisco Pizarro (lat 20.3'37'N, long 78'39'29'W). The second sentinel site was established in Córdoba department $(\mathrm{API}=6.2 ; \mathrm{AFI}=0.6)^{12}$ and the samples were collected in the localities of Tierralta (lat $8^{\circ} 10^{\prime} 34^{\prime \prime} \mathrm{N}$, long $76^{\circ} 03^{\prime} 46^{\prime \prime} \mathrm{W}$ ) and Montelíbano (lat 7 ${ }^{\circ} 59^{\prime} 13^{\prime \prime} \mathrm{N}$, long 75'25'30”W) and the third site, in San José (lat 2०34'15”N, long 72.38'25”W), department of Guaviare (API=6.4; AFI=0.9), ${ }^{12}$ where like Córdoba, there is a relatively low incidence of $P$. falciparum malaria but also little information about the behavior of the $P$. falciparum parasites in response to common antimalarials. The samples were collected in San José from November 2011 to May 2012, in Córdoba from July 2012 to June 2013, and in Nariño from February to June 2013. In Tumaco, the Afro-Colombian population predominates, whereas in Tierralta and Guaviare the populations are principally Mestizo and indigenous.

and verifying mono-infection for P. falciparum, $4 \mathrm{ml}$ of venous blood were taken from patients who agreed to participate voluntarily in the study. Blood samples were collected in EDTA tubes and distributed as follows: $200 \mu \mathrm{l}$ for microhematocrit, $400 \mu \mathrm{l}$ were spotted on Whatman grade 3 MM filter paper (Brentford, United Kingdom) for molecular study, and the remaining volume was used for ex vivo study.

The patients included in the study received standard treatment (day 0) according to the Colombian guide for antimalarial treatment for uncomplicated $P$. falciparum malaria. The treatment was 4 tablets twice a day for 3 days, with the number of tablets (each containing $20 \mathrm{mg}$ ART and $120 \mathrm{mg}$ LUM) dependent on weight. ${ }^{6}$ Patients were clinically supervised over 30 minutes by the medical staff after administration of the first dose to check for adverse reactions and then clinical and parasitological monitoring was conducted at days 1, 2, 3, 7, 14, 21 and 28. Endpoints considered in this study were completion of the follow-up period without treatment failure, loss to follow-up, withdrawal from study, protocol violation and treatment failure. At the end of 28 days, the patient response was evaluated according to the classification system of response to treatment as treatment success (adequate clinical and parasitological response-ACPR) and 
treatment failure (early treatment failure, late clinical failure, and late parasitological failure). ${ }^{6}$ On day 3 , the presence of parasites was verified by thick blood smear and PCR. ${ }^{14}$

\section{Ex vivo drug susceptibility assay}

Pre-dose culture plates with antimalarial drugs

Parasites cultures in 96-well plates were tested with four antimalarial drugs: chloroquine (CQ), LUM, ART and DHA (Sigma Aldrich, St. Louis, MO, USA) at the Colombian Instituto Nacional de Salud, following the methodology described by Aponte et al. ${ }^{10}$ For ART, a stock solution was made in ethanol. The concentration ranges of different drugs were 50-3200 nanomoles/ liter (nM) for CQ, 2.5-160 nM for LUM and 0.5-32 nM for ART and DHA. Drug plate quality control was performed under the same conditions used for samples, using the reference strains T4 (Thailand) $^{15}$ and 3D7 (BEI Resources, Manassas, VA, USA), ${ }^{16}$ which are resistant and sensitive to $C Q$ respectively. Afterwards, the drug plates were transported under refrigeration $\left(4-8^{\circ} \mathrm{C}\right)$ to sentinel sites, stored under the same conditions and used within two months of preparation.

\section{Mark III schizonts maturation method (microtest)}

Samples were processed at sentinel sites for a maximum of 6 hours after collection as previously described by Aponte et al., ${ }^{10}$ adjusting the parasitemia from 0.005 to $0.1 \%$ (250-5000 trophozoites/ $\mu$ l) with a $1.5 \%$ hematocrit. Two identical plates were prepared for each sample and incubated at $37^{\circ} \mathrm{C}$ in a standard atmosphere of $5 \% \mathrm{CO}_{2}, 5 \% \mathrm{O}_{2}$ and $90 \% \mathrm{~N}_{2}$. The microtest was stopped when the parasites reached at least $20 \%$ schizont maturation in the positive control well (without drugs). All wells were harvested as Giemsa-stained thick smears. The percentage of schizonts was calculated as the number of schizonts (with three or more nuclei) among 200 asexual forms using light microscopy $(1000 X){ }^{17}$ The $I_{50}$, defined as the drug concentration at which parasite maturation was $50 \%$ of that measured in drug-free control wells, was calculated using a non-linear regression model with the software $\mathrm{Hn}$-NonLin. ${ }^{18}$ Microtest reading quality control was performed at the central laboratory (supplementary data).

\section{Molecular markers}

Four genes were analyzed taking into account that single nucleotide polymorphisms and increased copy numbers in the Pfmdr1 gene have been linked to changes in the parasite susceptibility and therapeutic failure to mefloquine, artesunate, lumefantrine and quinine. ${ }^{19,20}$ Polymorphisms in Pfk13 (C580Y, Y493H and R539T) ${ }^{21}$ and Pfcrt (K76T) ${ }^{22}$ genes were associated with artemisinin and chloroquine resistance respectively, and the S769N Pfatp6 mutation was associated with raised artemether $\mathrm{IC}_{50}{ }^{23}$

\section{DNA extraction}

Plasmodium DNA was extracted from blood collected on filter paper employing a commercial kit (QIAamp DNA Micro Kit, Qiagen, Hilden, Germany) following the manufacturer's instructions.
Detection of S769N allele in gene Pfatp6 and K76T allele in gene Pfcrt

Allele detection was carried out by PCR-RFLP. The outer and nested PCR for gene Pfatp6 was performed following the methodology reported by Adhin et al. ${ }^{24}$ and Ferreira et al., ${ }^{25}$ respectively, and for gene Pfcrt using the methodology described by Londono et al. $^{22}$ (supplementary data). To determinate the allele in Pfatp6769, the digestion was made with the enzyme Afli (New England Biolabs, Ipswich, MA, USA), while for Pfcrt-76 ApoI (New England Biolabs) was used. The products of PCR-RFLP were separated in agarose gels, stained with ethidium bromide $(1 \mathrm{mg} / \mathrm{ml}$ ) and visualized in the image analyzer UV Gel Doc XR (software Quantity One 1-D, version 4.6.2, BioRad, Hercules, CA, USA).

\section{Sequencing of K13-propeller, Pfatp6 and Pfmdr1 genes}

Pfatp6 and Pfmdr1 genes were sequenced from field isolates with the highest and lowest $\mathrm{IC}_{50}$ for artemisinins (ART and DHA) and LUM, respectively. In order to determine polymorphisms in the K13-propeller gene, samples from day 0 (from patients who were followed until day 3) were amplified and sequenced following the methodology reported by Ménard et al. $^{26}$ The amplification of Pfatp6 and Pfmdr1 genes was performed according to Ferreira et al. $^{25}$ and Imwong et al., ${ }^{19}$ respectively. BioEdit Sequence Alignment Editor, version 7.2.5 (Ibis Biosciences, Carlsbad, CA, USA) and Codon Code Aligner, version 4.2.7, softwares were used for bioinformatic analysis. Polymorphisms associated with changes in susceptibility to antimalarials were evaluated in 14 positions of the Pfmdr1 gene and 29 of the Pfatp6 gene.

\section{Estimation of Pfmdr1 gene copy number}

Estimation of Pfmdr1 gene copy number was performed by realtime PCR using SYBR Green DyNAmo HS kit (Thermo Fisher Scientific Inc., Waltham, MA, USA). The primers used to amplify the Pfmdr1 gene were described by Ferreira et al. ${ }^{20}$ and the primers for the normalizer gene (myosin C: PfMyoC) were designed previously at the laboratory. The number of copies was determined by relative quantification using method $2^{-\Delta \Delta C t}, 20$ which assumes that both target (Pfmdr 1 ) and reference (PFMyo-C) genes are amplified with efficiencies near $100 \%$ and within $5 \%$ of each other. Once it was established that the target and reference genes have similar and nearly $100 \%$ amplification efficiencies, we determined the relative difference in expression level of the target gene in different samples. All assays were performed in duplicate in the thermocycler CFX96-BioRad detection system (BioRad, Hercules, CA, USA).

The primers' sequences and PCR programs for all molecular assays reported here, are summarized in the supplementary data.

\section{Statistical analysis}

All statistical analyses and generation of graphics were performed using Stata/SE software, version 12 (Stata Corporation, College Station, TX, USA). Ex vivo activity of each antimalarial drug was expressed as the geometric mean of inhibitory concentration $50\left(\mathrm{GM}^{-} \mathrm{IC}_{50}\right)$. The chi-square test was used to compare categorical variables. For all statistical tests, a p-value 
equal to 0.05 was deemed significant. Fisher's exact test (twotailed test) was used to analyze the statistical association between SNPs and drug sensitivity. The statistical association between Pfmdr1 copy number and sensitivity to LUM was obtained by correlation coefficients.

\section{Results}

\section{TES of artemether-lumefantrine combination}

Of the 150 patients enrolled (Figure 2), only 49 (32.7\%) completed the clinical and parasitological follow-up at 28 days; from these 69.4\% (34/49) were men, 69.4\% (34/49) were AfroColombians and $77.6 \%$ (38/49) were from urban areas in the municipalities studied. The median age was 25 years, ranging between seven and 75 years old, and 63.3\% (31/49) of patients were older than 20 years of age. On day 0 the range of parasitemia was between 70 and 54800 trophozoites/ $\mu$ with an average of 5880 trophozoites/ $\mu$ l, and seven patients (14.3\%) had gametocytes. Due to the reduction of malaria cases, a patient with parasitemia greater than 50000 trophozoites/ $\mu$ l was included; this individual did not show any signs of complicated malaria and had strict medical supervision. The distributions of the above variables, according to the locality are shown in Table 1.

The most common symptoms were: headache 98\% (48/49); chills $91.8 \%$ (45/49); sweating $85.7 \%$ (42/49); myalgias $85.7 \%$ (42/49); anorexia 83.7\% (41/49); fever 77.6\% (38/49). Hematocrit values ranged between 28 and $48 \%$, with an average of $37.4 \%$ and a median of $37 \%$.

On physical examination $12.2 \%$ (6/49) patients had hepatomegaly and $14.3 \%$ (7/49) splenomegaly ( $1 \mathrm{~cm}$ below the costal margin left/right, respectively). All patients had negative count for asexual parasites on the second day of monitoring, while sexual parasitemia persisted until day 7 , and the headache, chills and fever until day 14 , day 3 and day 1 , respectively (Table 1). The final outcome at 28 days of follow-up in these 49 patients was classified as ACPR (i.e. clinical and parasitological resolution by the end of the follow-up period). No serious adverse events were observed after the administration of the first dose.

\section{Evaluation of parasitemia at day 3 post-treatment}

Ninety out of the 150 (60\%) patients included in the study had clinical and parasitological follow-up during days 1,2 and 3 . Fifty four of them (60\%) cleared the asexual parasitaemia at 24 hours and the remaining $40 \%(36 / 90)$ at 48 hours. PCR correction was made in $78 / 90$ (86.6\%) samples taken on day 3 with negative results, except for two samples from Tumaco, which were positive for $P$. falciparum. This result can be explained by the presence of gametocytes in the thick blood smear.

\section{Drug susceptibility assay}

A total of $111 P$. falciparum isolates included on day 0 were processed by microtest (Figure 2). Only one (0.9\%) of these samples (from Tumaco) did not achieve the required percentage of schizont maturation and another sample was not evaluated for chloroquine due to a technical failure. Geometric mean (GM) (95\% CI) of the IC $_{50}$ of the total samples analyzed for each of the drugs was $141.15 \mathrm{nM}$ for CQ (95\% CI 125.26 to 159.06), $7.07 \mathrm{nM}$ for LUM (95\% CI 6.29 to 7.96$), 1.65 \mathrm{nM}$ for ART (95\% CI 1.44 to 1.91 ) and $1.18 \mathrm{nM}$ for $\mathrm{DHA}$ (95\% CI 1.03 to 1.35 ). Overall, the results of the three sentinel sites showed low susceptibility to CQ in $64.2 \%$ (70/109) of isolates (Figure 3). Although the studied populations have very good in vitro susceptibility to LUM, some of the analyzed isolates had a slight decrease in susceptibility. Of the 110 isolates tested, 5.4\% (6/ 110) had $I_{50}>20 \mathrm{nM}$ for LUM, one of which came from Nariño, two from Guaviare and three from Córdoba (Figure 3). In the case of ART and DHA, over $90 \%$ of isolates had $\mathrm{IC}_{50}<5 \mathrm{nM}$ in both cases (ART=91.9\% and $\mathrm{DHA}=95.5 \%$ ) (Figure 3). $\mathrm{GMIC}_{50}$ for CQ was greater than $100 \mathrm{nM}$ in the departments of Córdoba and Nariño, being significantly higher in parasites isolated from

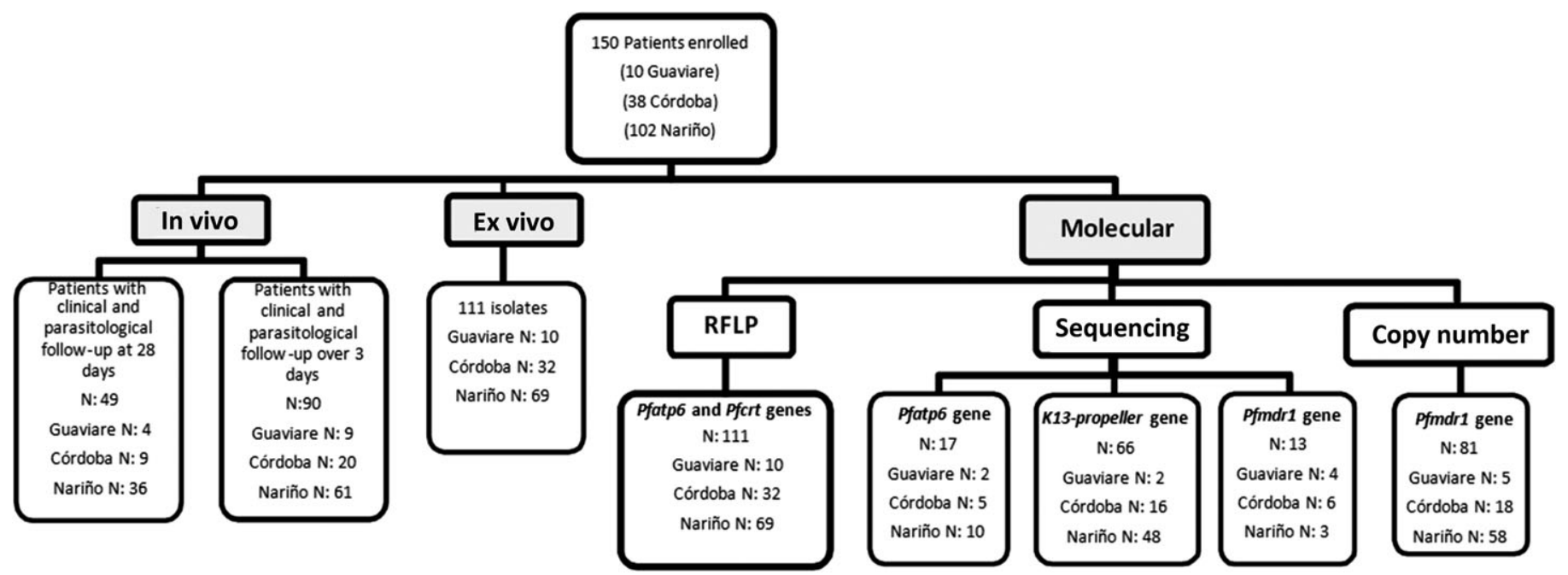

Figure 2. Flowchart describing the number of samples tested in each of the three studies. Patients enrolled and number of samples tested in the in vivo, ex vivo and molecular components of this study. 
Table 1. General demographic characteristics of the population included in the study of therapeutic efficacy and parasitological follow-up

\begin{tabular}{|c|c|c|c|c|}
\hline Characteristics & \multicolumn{4}{|l|}{ Study sites } \\
\hline n (\%) $[95 \% \mathrm{CI}]$ & $4 / 49$ (8.2) [2.3 to 19.6$]$ & $2 / 49(4)[0.5$ to 14$]$ & $7 / 49$ (14) [6 to 27.2] & $36 / 49$ (73) [58.9 to 85.1$]$ \\
\hline Proportion of men (\%) [95\% CI] & $2 / 4$ (50) [6.8 to 93.2$]$ & $1 / 2(50)$ [1.3 to 98.7$]$ & $6 / 7$ (86) [42.1 to 99.6$]$ & $25 / 36$ (69) [51.9 to 83.7] \\
\hline Mestizo & 4/4 (100) [39.8 to 100$]$ & $1 / 2(50)[1.3$ to 98.7$]$ & $2 / 7$ (29) [3.7 to 71$]$ & $7 / 36$ (19) $[8.2$ to 36$]$ \\
\hline Afro-Colombian & 0 & $1 / 2(50)[1.3$ to 98.7$]$ & $5 / 7(71)[29$ to 96.3$]$ & $28 / 36$ (78) [60.8 to 90$]$ \\
\hline $\begin{array}{l}\text { Asexual parasitemia average on } \\
\text { day } 0\end{array}$ & 3566 & 17007 & 4148 & 5856 \\
\hline $\begin{array}{l}\text { Patients with gametocytaemia } \\
\text { on day } 0: \mathrm{n}(\%)\end{array}$ & $2 / 4(50)$ & $1 / 2(50)$ & $1 / 7(14)$ & 4/36 (11) \\
\hline Day 2 & $3 / 3(100)$ & $2 / 2(100)$ & $3 / 3(100)$ & $10 / 10(100)$ \\
\hline \multicolumn{5}{|l|}{ Persistence of gametocytes (n) } \\
\hline Day 1 & 1 & NA & NA & NA \\
\hline Day 2 & 1 & NA & NA & NA \\
\hline Day 3 & NA & 1 & NA & $3^{a}$ \\
\hline Day 7 & NA & NA & 1 & 1 \\
\hline
\end{tabular}

Nariño ( $p=0.02$ ), whereas in Guaviare $P$. falciparum populations susceptible to CQ were found (Table 2). In contrast with the results for $C Q$, the $\mathrm{IC}_{50}$ for $L U M$ did not differ significantly among departments.

\section{Molecular markers}

Detection of S769N allele in Pfatp6 and K76T allele in Pfcrt

All isolates had the wild-type allele 5769 in Pfatp6, while in Pfcrt all isolates were found to be mutants (76T).

Detection of SNPs in the K13-propeller, Pfatp6 and Pfmdr1 genes For sequencing Pfatp6, K13-propeller and Pfmdr1 genes, 17, 66 and 13 samples were included, respectively (Figure 2). No polymorphism was found in the K13-propeller gene in the region coding for the propeller domain. For Pfmdr1 13 isolates were sequenced, six with LUM IC 50 above $20 \mathrm{nM}(21.45 \mathrm{nM}$ to $50.37 \mathrm{nM})$ and seven with the lowest $\mathrm{IC}_{50}(1.56 \mathrm{nM}$ to $5.11 \mathrm{nM})$. Fourteen polymorphisms previously reported were analyzed, the D1246Y mutation was found in all isolates from Guaviare and
Córdoba, but it was not detected in Nariño isolates, while Y184F and N1042D polymorphisms and the deletion of a repetition ATT (located in a region of low complexity of the gene) were found in all isolates analyzed. Additionally, a non-synonymous mutation G406A was identified in one isolate from Córdoba with a LUM IC 50 of $24.3 \mathrm{nM}$ (Table 3 ).

For Pfatp6 the eight isolates that had $\mathrm{IC}_{50}>6 \mathrm{~nm}$ to ART or DHA $(6.33 \mathrm{nM}$ to $12.17 \mathrm{nM})$ and nine isolates that had the lowest $\mathrm{IC}_{50}$ for these drugs $(0.5 \mathrm{nM}$ to $3.48 \mathrm{nM})$ were chosen. Twenty nine polymorphisms previously reported were analyzed, only one synonymous mutation in the I898I position and the deletion of codon 847 were identified in all samples (Table 4). In addition, three polymorphisms were identified, which have not been reported previously: a deletion of six bases ranging from position 2350 to 2355 and two non-synonymous mutations at positions S466N and V307D, the latter present in 37.5\% (3/8) of the samples had the highest $\mathrm{IC}_{50}$ for ART or DHA.

\section{Pfmdr1 copy number estimation}

Eighty one out of the $111(73.0 \%)$ isolates were analyzable by qPCR: $68 \%$ of them (55/81) had a single copy of the gene, $17 \%$ 

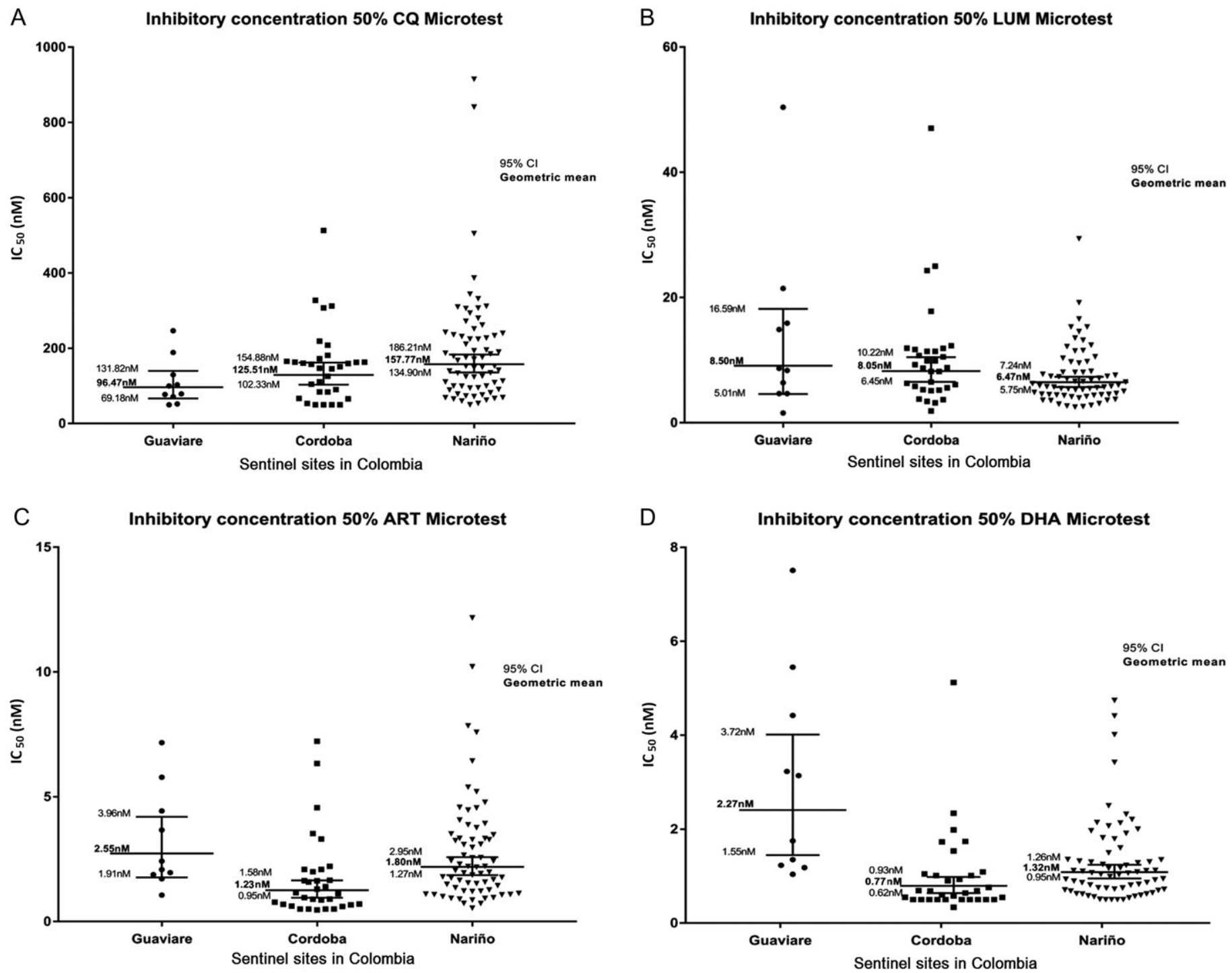

Figure 3. Ex vivo susceptibility of $P$. falciparum isolates to four antimalarial drugs, stratified by sentinel sites. The susceptibility of the parasites to A. chloroquine (CQ), B. lumefantrine (LUM), C. artemether (ART) and D. dihydroartemisinin (DHA) was determined by the schizonts maturation method Microtest.

(14/81) presented two copies, 10\% (8/81) three copies and $2.5 \%(2 / 81)$ four and five copies (Figure 4).

\section{Discussion}

Therapeutic efficiency study results demonstrated the efficacy and safety of the ART-LUM combination in the studied populations, a situation that had been reported previously in Colombia for other $\mathrm{ACT}^{7,8}$ It is worth mentioning that reaching the sample size was quite difficult, due to the drastic reduction of cases of P. falciparum malaria that occurred between 2011 and 2012, which was $58 \%$ and $85 \%$ for Córdoba and Guaviare, respectively. ${ }^{12}$ To improve patient recruitment, several strategies were implemented, such as including individuals with parasitemia levels below 250 trophozoites/ $\mu$ l, carrying out active search for patients, extending the period of inclusion, and increasing the number of locations. In addition, the high number of patients lost during follow-up contributed to not achieving an adequate sample size for TES.

The above facts demonstrate the need to implement a shorter epidemiologic strategy, such as the clinical and parasitological surveillance at day 3 post-treatment, considering that artemisinin provides a particular advantage over other treatments: rapid parasite clearance, which was demonstrated by the absence of parasites by thick blood smears in $97 \%$ of patients with parasitemia between 10000 and 100000 parasites/ $\mu$ l on day 3 post-treatment. ${ }^{5}$ Our work showed that on day 2 , no patient had asexual positive parasitemia, which allows us to recommend the implementation of clinical and parasitological follow-up at day 2 and 3 post-treatment, in order to detect early parasite clearance delays. This surveillance can be 
Table 2. Ex vivo susceptibility of $P$. falciparum isolates from three sentinel sites in Colombia to four antimalarial drugs

\begin{tabular}{|c|c|c|c|c|c|c|}
\hline \multirow[t]{3}{*}{ Antimalarial Drug } & \multicolumn{2}{|c|}{ Guaviare } & \multicolumn{2}{|c|}{ Cordoba } & \multicolumn{2}{|c|}{ Nariño } \\
\hline & \multicolumn{2}{|c|}{$\mathrm{GMIC}_{50}$ (range) } & \multicolumn{2}{|c|}{$\mathrm{GMIC}_{50}$ (range) } & \multicolumn{2}{|c|}{$\mathrm{GMIC}_{50}$ (range) } \\
\hline & $\mathrm{n}$ & $\mathrm{nM}$ & $n$ & $\mathrm{nM}$ & $n$ & $\mathrm{nM}$ \\
\hline Chloroquine & 10 & $96.47(50-246.36)$ & 32 & $125.51(50-512.98)$ & 68 & $157.77(50-914.84)$ \\
\hline Lumefantrine & 10 & $8.50(1.56-50.37)$ & 33 & $8.05(1.8-47.03)$ & 68 & $6.47(2.5-29.38)$ \\
\hline Artemether & 10 & $2.55(1.06-7.17)$ & 33 & $1.23(0.47-7.23)$ & 68 & $1.80(0.5-12.17)$ \\
\hline Dihydroartemisin & 10 & $2.27(0.88-7.51)$ & 33 & $0.77(0.27-5.12)$ & 68 & $1.32(0.5-7.58)$ \\
\hline
\end{tabular}

$\mathrm{IC}_{50}$ : concentration of drug that is required for $50 \%$ inhibition in vitro; $\mathrm{GMIC}_{50}$ : geometric mean of $\mathrm{IC}_{50}$; $\mathrm{n}$ : number of isolates tested; nM: nanomoles/liter.

Table 3. Polymorphisms found in the Pfmdr1 gene in 13 Colombian isolates

\begin{tabular}{|c|c|c|c|c|}
\hline Polymorphism & Codon & Nucleotide position & Allele: frequency n (\%) & References \\
\hline G406A & $\mathbf{G}(\mathrm{GGA}) / \mathbf{A}(\mathrm{GCA})$ & G1217C & $\begin{array}{l}\text { G: } 12(92) \\
\text { A: } 1 \text { sample from TA (8) }\end{array}$ & $\begin{array}{l}\text { Polymorphism not } \\
\text { reported previously }\end{array}$ \\
\hline N86Y & $N(A A T) / Y(T A T)$ & $\mathrm{A} 256 \mathrm{~T}$ & $\mathrm{~N}: 13(100)$ & Grobusch et al. ${ }^{27}$ \\
\hline E130K & $E(G A A) / K(A A A)$ & G388A & $\mathrm{E}: 13(100)$ & Veiga et al. ${ }^{28}$ \\
\hline Y184F & $\mathbf{Y}(\mathrm{TAT}) / \mathbf{F}(\mathrm{TTT})$ & A553T & $\mathrm{F}: 13(100)$ & Veiga et al. ${ }^{28}$ \\
\hline G249 & $\mathrm{G}(\mathrm{GGA} / \mathrm{GGG})$ & A747G & G: $13(100)$ & Veiga et al. ${ }^{28}$ \\
\hline $\mathrm{L} 327 \mathrm{H}$ & $\mathrm{L}(\mathrm{CTT}) / \mathrm{H}(\mathrm{CAT})$ & T980A & L: $13(100)$ & Imwong et al. ${ }^{19}$ \\
\hline D587E & $\mathrm{D}(\mathrm{GAT}) / \mathrm{E}(\mathrm{GAA})$ & $\mathrm{T} 1780 \mathrm{~A}$ & D: $13(100)$ & Imwong et al. ${ }^{19}$ \\
\hline N660 (deletion) & $\mathbf{N}(\mathrm{ATT})$ & $1978-1980$ & Deletion: 13 (100) & Veiga et al. ${ }^{28}$ \\
\hline A750T & $\mathrm{A}(\mathrm{GCA}) / \mathrm{T}(\mathrm{ACA})$ & $\mathrm{G} 2248 \mathrm{~A}$ & A: $13(100)$ & Veiga et al. ${ }^{28}$ \\
\hline S784L & $\mathrm{S}(\mathrm{TCA}) / \mathrm{L}(\mathrm{TTA})$ & C2350T & S: $13(100)$ & Veiga et al. ${ }^{28}$ \\
\hline S1034C & $\mathrm{S}(\mathrm{AGT}) / \mathrm{C}(\mathrm{TGC})$ & A3106T & S: $13(100)$ & Reed et al. ${ }^{29}$ \\
\hline N1042D & $\mathbf{N}(A A T) / D(G A T)$ & A3124G & D: 13 (100) & Reed et al. ${ }^{29}$ \\
\hline F1226Y & $\mathrm{F}(\mathrm{TTT}) / \mathrm{Y}(\mathrm{TAT})$ & T3677A & $\mathrm{F}: 13(100)$ & Veiga et al. ${ }^{28}$ \\
\hline D1246Y & $\mathbf{D}(\mathrm{GAT}) / \mathbf{Y}(\mathrm{TAT})$ & G3735T & $\begin{array}{l}\text { D: } 3 \text { samples from TUM (23) } \\
\text { Y: } 10 \text { (77) }\end{array}$ & Reed et al. ${ }^{29}$ \\
\hline
\end{tabular}

TA: Tierralta (Córdoba department); TUM: Tumaco (Nariño department). Polymorphisms with mutations when detected in the analyzed isolates, have been highlighted in bold.

routinely done by the national diagnostic network and would be the simplest and fastest way to detect early changes in the susceptibility of the parasite to artemisinins. These results would be the basis for proposing a TES, which will provide evidence for therapeutic failure.

On the other hand, the percentage of sexual forms in the population was nearly $20 \%$, and Tumaco was the locality with greater persistence of gametocytes post-treatment. Although artemisinin has gametocytocidal effect on the early stages (I-III), it does not prevent the transmission of the parasite to the mosquito. ${ }^{34}$ This may suggest the common existence of long-term infections and the need for the addition of gametocytocidal drugs such as primaquine in the ACT scheme, as a strategy to preserve the effectiveness of combination therapies and to eliminate the likelihood of reservoirs. A previous epidemiological study in Colombia highlighted the importance of using primaquine, because the elimination of gametocytes is much faster.

In the ex vivo study, the evaluated isolates show high susceptibility to ART, LUM and DHA. However, it is noteworthy that for LUM, $27.2 \%(30 / 110)$ of the isolates had $\mathrm{IC}_{50}>10 \mathrm{nM}$, of which $1.8 \%(2 /$ 110) were higher than $40 \mathrm{nM}$ compared to the results obtained by Aponte et al. in 2006 and 2007, where the IC 50 for LUM and ART was less than $10 \mathrm{nM}$ for all isolates tested. ${ }^{10}$ In the case of artemisinin derivatives, it was observed that more than $90 \%$ of isolates had an $\mathrm{IC}_{50}<5 \mathrm{nM}$ and only $1.8 \%(2 / 110)$ showed $\mathrm{IC}_{50}$ of $12.17 \mathrm{nM}$ and $10.21 \mathrm{nM}$ for ART. These changes in susceptibility in vitro of some isolates, which still have low $\mathrm{IC}_{50}$ according to the previously reported 
Table 4. Analysis of polymorphisms in the Pfatp6 gene in 17 Colombian isolates

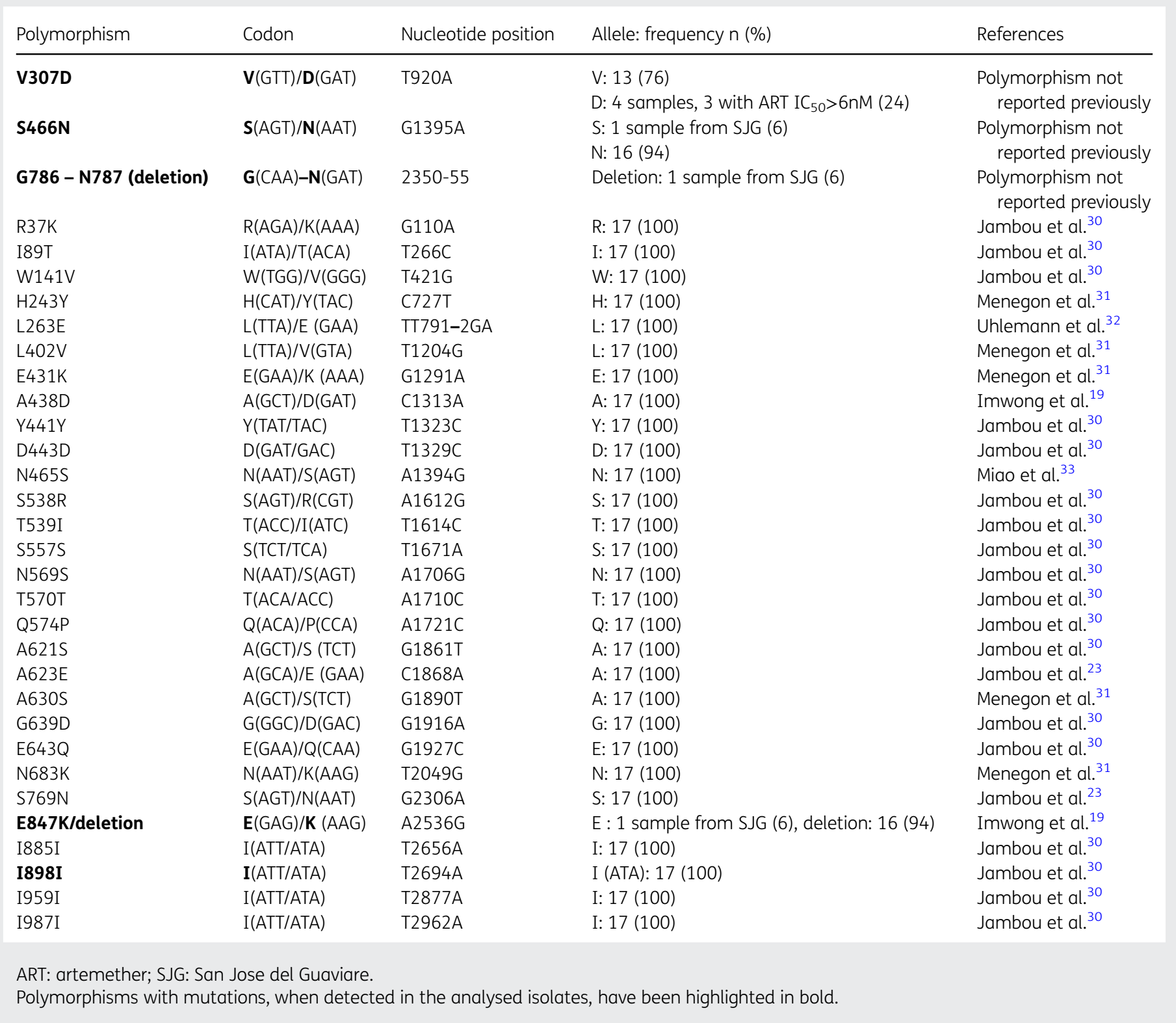

thresholds related to resistance, ${ }^{23}$ reveal the importance of carefully monitoring the in vitro response of parasite populations for artemisinin and the partner drug, in order to enable timely identification of changes that compromise such therapies. Recently, it was shown that the ring-stage survival assay (RSA) $)^{35}$ can assess more accurately in vitro behavior of parasites compared with conventional tests, because it measures the survival rate of parasites in stage ring to a short pulse with DHA (the active metabolite of artemisinin). One of the limitations of the present study is the lack of information regarding RSA, since this methodology was not available during the development of the work. We are aware of the need to implement this new trial in the country for routine surveillance and future resistance studies.
Although chloroquine is not part of current therapeutic regimens for $P$. falciparum malaria, it was used in Colombia until 2002. Interestingly, we observed an increase in the in vitro susceptibility to CQ of isolates circulating in Tumaco, in comparison with isolates evaluated previously. ${ }^{10}$ Thus, the GMIC $_{50}$ to $\mathrm{CQ}$ using microtest in this locality was much higher previously $(2006 / 2007=319.2 / 341.5$ nM vs 2013=157.77 nM). This observation suggests a re-expansion of wild-type parasite populations compared with mutant parasites (K76T) in response to the absence of $C Q$ pressure. However, all isolates with $\mathrm{IC}_{50}$ for $\mathrm{CQ}$ higher or lower than $100 \mathrm{nM}$ had the 76T mutation in the Pfcrt gene, indicating not only fixation of alleles but the existence of other mutations in this gene or in other genes, which may be 


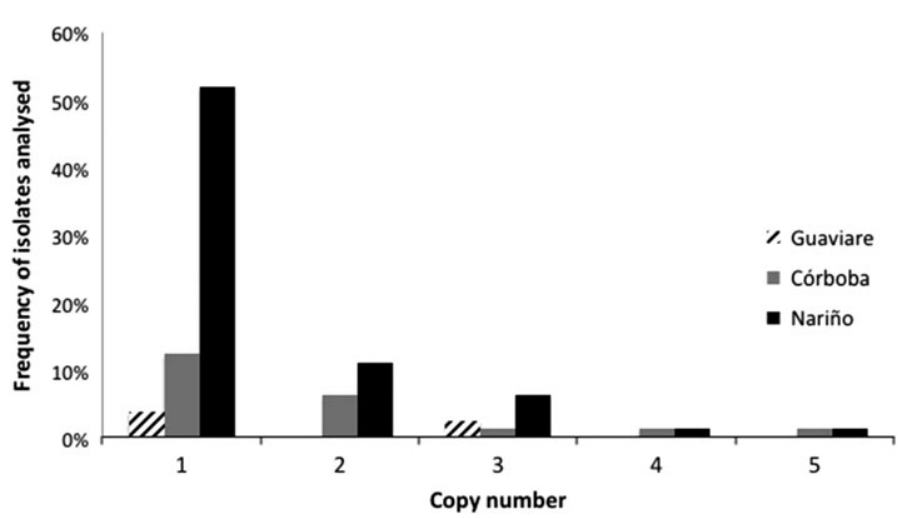

Figure 4. Pfmdr1 copy number estimation in Plasmodium falciparum isolates collected from the three sentinel sites.

re-establishing susceptibility to the drug in an adaptive manner. This has previously been demonstrated in isolates from French Guiana, where the C350R mutation in Pfcrt is directly correlated with an increased susceptibility to CQ. ${ }^{36}$

Polymorphisms found in Pfmdr1 and Pfatp6 had no significant correlation ( $p>0.05$ ) with the increase or decrease of the $\mathrm{IC}_{50}$ for the drugs evaluated. A previous study with 15 isolates from different areas of the Colombian Pacific evaluated both SNPs and copy numbers in Pfmdr1, finding only one copy of the gene. ${ }^{37}$ In our study, we found that $32 \%$ (26/81) of the samples had more than one copy of Pfmdr1. Nevertheless, a significant correlation between copy number and $\mathrm{IC}_{50}$ values for the drugs tested was not found. The increase in the number of copies of Pfmdr1 was initially described in reference strains resistant to $C Q$ and $M Q$ as $\mathrm{W} 2^{38}$ or clones of the Dd2 strain ${ }^{39}$ and is correlated widely with low susceptibility to mefloquine. However, despite the fact that lumefantrine belongs to the same group of arylamino alcohols as mefloquine, no significant correlation was found, suggesting a different mechanism of action with mefloquine. Additionally, it is likely that a greater copy number of Pfmdr1 $(\geq 5)$ is needed to find a correlation with higher $\mathrm{IC}_{50}$ for DHA, as was demonstrated by Cui et al. ${ }^{39}$ Ariey et al. found mutations in the K13-propeller gene strongly associated with the response to artemisinin, ${ }^{21}$ which were evaluated in this study. None of the previously reported mutations were found, which correlates with the adequate therapeutic response and rapid parasite clearance.

In conclusion, the artemether-lumefantrine combination in the three studied localities showed a high efficacy for the treatment of uncomplicated $P$. falciparum malaria. This clinical outcome, along with parasite clearance times on the second day post-treatment and presence of parasites with wild-type genotype for the K13-propeller gene, constitutes the susceptibility baseline that will serve as the starting point for futures studies to systematically monitor changes in the behavior of parasites to the current first-line treatment scheme for $P$. falciparum malaria in Colombia.

\section{Supplementary data}

Supplementary data are available at Transactions Online (http:// trstmh.oxfordjournals.org/).
Authors' contributions: SA and APG conceived the study; SA, CA-L, CR and MFY implemented the study in the field; CR carried out the therapeutic efficacy study; SA and CA-L performed the ex vivo testing; SDB and CG carried out the molecular study; CA-L, SDB, SA, CR, APG, CG and AK-O performed statistical analysis and interpretation of data; SA, APG, $\mathrm{SDB}$ and $\mathrm{AK}-\mathrm{O}$ critically revised the manuscript for intellectual content. All of the authors read and approved the final manuscript. SA and APG are guarantors of the paper.

Acknowledgements: We would like to thank Zulma Cucunubá MD, for the design of the therapeutic efficacy study; the communities in San José del Guaviare, Tierralta, Montelíbano, Francisco Pizarro and Tumaco; the Secretariats of Public Health and Vector Control Programs of these zones; the hospitals of San José and Tumaco, and finally, the Parasitology Group for providing the rapid malaria diagnosis tests.

Funding: This work was supported by COLCIENCIAS [project 210451929111] and the Instituto Nacional de Salud. Funding organizations had no role in study design, data collection and analysis, decision to publish or preparation of the manuscript.

Competing interests: None declared.

Ethical approval: According to national and international standards, the study was approved by the Ethics Committee of the Instituto Nacional de Salud de Colombia [minutes of approval \#9 on October 7, 2010]. Informed consent was obtained from the patients, who agreed to participate in the study; for underage patients, parental written consent was provided. All the results obtained were officially delivered and explained to the health authorities. The patients received anti-malarial treatment according to the national guidelines at the time of the study.

\section{References}

1 WHO. Global Technical Strategy for Malaria 2016-2030. Geneva: World Health Organization; 2015. http://www.who.int/malaria/areas/ global_technical_strategy/en/ [accessed 01 February 2016].

2 Denis MB, Tsuyuoka R, Poravuth Y et al. Surveillance of the efficacy of artesunate and mefloquine combination for the treatment of uncomplicated falciparum malaria in Cambodia. Trop Med Int Health 2006;11:1360-6.

3 Red Amazónica de Vigilancia de la Resistencia a las Drogas Antimaláricas/Amazon Malaria Iniciative (RAVREDA/AMI)-OPS. Guía práctica para estudios in vivo de eficacia de los medicamentos antimaláricos en las Américas. Iquitos, Perú. 2003. http://www.paho.org/ hq/index.php?option=com_docman\&task=doc_download\&Itemid $=270 \&$ gid=18303\&lang=es [article in Spanish; accessed 01 February 2016].

4 Fairhurst RM, Nayyar GM, Breman JG et al. Artemisinin-resistant malaria: research challenges, opportunities, and public health implications. Am J Trop Med Hyg 2012;87:231-41.

5 Stepniewska K, Ashley E, Lee SJ et al. In vivo parasitological measures of artemisinin susceptibility. J Infect Dis 2010;201:570-9.

6 Ministerio de Protección Social/OPS. Guía de atención clínica de malaria. Instituto Nacional de Salud, Bogotá, Colombia; 2010. http://www. paho.org/col/index.php?option=com_docman\&task=doc_download\&gid= 1220\&Itemid=. [article in Spanish; accessed 25 January 2016].

7 Vásquez A, Sanín F, Álvarez LG et al. Therapeutic efficacy of a regimen of artesunate-mefloquine-primaquine treatment for Plasmodium falciparum malaria and treatment effects on gametocytic development. Biomédica 2009;29:307-19. [article in Spanish]. 


\section{S. Aponte et al.}

8 De la Hoz F, Porras A, Rico A et al. Artesunate + amodiaquine versus artemether-lumefantrine for the treatment of uncomplicated Plasmodium falciparum malaria in the Colombian Pacific region: a non-inferiority trial. Rev Soc Bras Med Trop 2012;45:732-8.

9 Arango E, Carmona-Fonseca J, Blair S. In vitro susceptibility of Colombian Plasmodium falciparum isolates to different antimalarial drugs. Biomédica 2008;28:213-23. [article in Spanish]

10 Aponte SL, Díaz G, Pava Z et al. Sentinel network for monitoring in vitro susceptibility of Plasmodium falciparum to antimalarial drugs in Colombia: a proof of concept. Mem Inst Oswaldo Cruz 2011;106:123-9.

11 Instituto Nacional de Salud. Informe Epidemiológico Nacional 2013. Enfermedades transmitidas por vectores: malaria. http://www.ins.gov. co/lineas-de-accion/Subdireccion-Vigilancia/Informe\%20de\%20Evento\% 20Epidemiolgico/MALARIA\%202013.pdf [article in Spanish; accessed 10 January 2016].

12 Instituto Nacional de Salud. Informe Epidemiológico Nacional 2012. Enfermedades transmitidas por vectores: malaria. http://www.ins.gov. co/lineas-de-accion/Subdireccion-Vigilancia/Informe\%20de\%20Evento\% 20Epidemiolgico/MALARIA\%202012.pdf [article in Spanish; accessed 10 January 2016].

13 Mount DL, Nahlen BL, Patchen LC, Churchill FC. Adaptations of the Saker-Solomons test: simple, reliable colorimetric field assays for chloroquine and its metabolites in urine. Bull World Health Organ 1989;67:295-300.

14 Snounou G,Viriyakosol S, Zhu XP et al. High sensitivity of detection of human malaria parasites by the use of nested polymerase chain reaction. Mol Biochem Parasitol 1993;61:315-20.

15 Chaparro J, Wasserman M. Adecuación de una prueba radiométrica para la detección de resistencia múltiple de Plasmodium falciparum a medicamentos. Biomédica 1999;19:25-34. [article in Spanish]

16 BEI Resources Repository, NIAID, NIH: Plasmodium falciparum, Strain 3D7, MRA-102, contributed by Daniel J. Carucci. https://www. beiresources.org/Catalog/BEIParasiticProtozoa/MRA-102.aspx [accessed 30 March 2017].

17 WHO. In vitro micro-test (Mark III) for the assessment of the response of Plasmodium falciparum to chloroquine, mefloquine, quinine, amodiaquine, sulfadoxine/pyrimethamine and artemisinin. Geneva: World Health Organization; 2001. http://www.who.int/ malaria/publications/atoz/markiii.pdf [accessed 25 January 2011].

18 Noedl H, Wernsdorfer WH, Miller RS, Wongsrichanalai C. Histidine-rich protein II: a novel approach to malaria drug sensitivity testing. Antimicrob Agents Chemother 2002;46:1658-64.

19 Imwong M, Dondorp AM, Nosten F et al. Exploring the contribution of candidate genes to artemisinin resistance in Plasmodium falciparum. Antimicrob Agents Chemother 2010;54:2886-92.

20 Ferreira I, Do Rosário V, Cravo P. Real-time quantitative PCR with SYBR Green I detection for estimating copy numbers of nine drug resistance candidate genes in Plasmodium falciparum. Malar J 2006;5:1-6.

21 Ariey F, Witkowski B, Amaratunga $C$ et al. A molecular marker of artemisinin-resistant Plasmodium falciparum malaria. Nature 2014; 505:50-5.

22 Londono B, Eisele TP, Keating J et al. Chloroquine-resistant haplotype Plasmodium falciparum parasites, Haiti. Emerg Infect Dis 2009;15: 735-40.

23 Jambou R, Legrand E, Niang $M$ et al. Resistance of Plasmodium falciparum field isolates to in-vitro artemether and point mutations of the SERCA-type PfATPase6. Lancet 2005;366:1960-3.
24 Adhin M, Labadie-Bracho M, Vreden SG. Status of potential PfATP6 molecular markers for artemisinin resistance in Suriname. Malar J 2012;11:322.

25 Ferreira I, Martinelli A, Rodrigues LA et al. Plasmodium falciparum from Pará state (Brazil) shows satisfactory in vitro response to artemisinin derivatives and absence of the S769N mutation in the SERCA-type PfATPase6. Trop Med Int Health 2008;13:199-207.

26 Ménard D, Khim N, Ariey F, Langlois A. PCR and sequencing for genotyping of candidate Plasmodium falciparum artemisinin resistance SNPs in the Kelch 13 gene v1.0. Institute Pasteur du Cambodge; 2013. http://www.wwarn.org/tools-resources/procedures/pcr-and-sequencinggenotyping-candidate-plasmodium-falciparum-artemisinin [accessed 25 January 2015].

27 Grobusch MP, Adagu IS, Kremsner PG, Warhurst DC. Plasmodium falciparum: in vitro chloroquine susceptibility and allele-specific PCR detection of Pfmdr1 Asn86Tyr polymorphism in Lambarene, Gabon. Parasitology 1998;116:211-7.

28 Veiga MI, Ferreira PE, Jörnhagen $L$ et al. Novel polymorphisms in Plasmodium falciparum $\mathrm{ABC}$ transporter genes are associated with major ACT antimalarial drug resistance. PLoS One 2011;6:e20212.

29 Reed MB, Saliba KJ, Caruana SR et al. Pgh1 modulates sensitivity and resistance to multiple antimalarials in Plasmodium falciparum. Nature 2000;403:906-9.

30 Jambou R, Martinelli A, Pinto J et al. Geographic structuring of the Plasmodium falciparum sarco(endo)plasmic reticulum Ca2+ ATPase (PfSERCA) gene diversity. PLoS One 2010;5:e9424.

31 Menegon M, Talha AA, Severini C et al. Frequency distribution of antimalarial drug resistance alleles among Plasmodium falciparum isolates from Gezira State, central Sudan, and Gedarif State, eastern Sudan. Am J Trop Med Hyg 2010;83:250-57.

32 Uhlemann AC, Cameron A, Eckstein-Ludwig $U$ et al. A single amino acid residue can determine the sensitivity of SERCAs to artemisinins. Nat Struct Mol Biol 2005;12:628-9.

33 Miao M, Wang Z, Yang Z et al. Genetic diversity and lack of artemisinin selection signature on the Plasmodium falciparum ATP6 in the Greater Mekong Subregion. PLoS One 2013;8:e59192.

34 Djimde AA, Maiga AW, Ouologuem D et al. Gametocyte clearance dynamics following oral artesunate treatment of uncomplicated falciparum malaria in Malian children. Parasite 2016;23:3.

35 Witkowski B, Amaratunga C, Khim N et al. Novel phenotypic assays for the detection of artemisinin-resistant Plasmodium falciparum malaria in Cambodia: in-vitro and ex-vivo drug-response studies. Lancet Infect Dis 2013;13:1043-9.

36 Pelleau S, Moss EL, Dhingra SK et al. Adaptive evolution of malaria parasites in French Guiana: Reversal of chloroquine resistance by acquisition of a mutation in Pfcrt. Proc Natl Acad Sci U S A 2015;112: 11672-7.

37 Echeverry DF, Holmgren G, Murillo C et al. Short report: polymorphisms in the Pfcrt and Pfmdr1 genes of Plasmodium falciparum and in vitro susceptibility to amodiaquine and desethylamodiaquine. Am J Trop Med Hyg 2007;77:1034-8.

38 Wilson CM, Serrano AE, Wasley A et al. Amplification of a gene related to mammalian mdr genes in drug-resistant Plasmodium falciparum. Science 1989;244:1184-6.

39 Cui L, Wang Z, Miao J et al. Mechanisms of in vitro resistance to dihydroartemisinin in Plasmodium falciparum. Molecular Microbiol 2012; 86:111-28. 